\title{
CALIFICACIONES, PRUEBAS OBJETIVAS Y APRENDIZAJE SIGNIFICATIVO EN QUÍMICA Y FÍSICA DE COU
}

\author{
VÁZQUEZ ALONSO, A.
}

Servicio de Inspección Técnica de Educación. C/ San Miguel, 28, 1‥07002 Palma de Mallorca (Baleares).

\section{SUMMARY}

Herein are studied the relations between Chemistry and Physics school marks in COU and in the first university year, and the score obtained at an objective test designed on the COU curriculum. The output of correlations is low and the analysis of the regression shows that the predictive ability of the variables of the objective test with respect to the marks is small; paradoxically, it is bigger for the marks at university than for those in COU, whose curriculum was the base for the test. Besides, predictive results are higher for Chemistry than for Physics. Non-significant learning could explain both, low results and the differences observed.

Desde hace tiempo, las críticas sobre las deficiencias de las calificaciones escolares para describir con validez y fiabilidad el dominio o maestría alcanzado por los alumnos, como consecuencia de la instrucción, ha sido un tema habitual en la docimología y en las investigaciones sobre las evaluaciones escolares. El empleo de pruebas objetivas (POs), o tests de rendimiento, constituye la alternativa más fiable y válida a la evaluación tradicional de los alumnos cuyas principales ventajas son la estandarización / objetividad (independencia de la puntuación respecto a las situaciones y los evaluadores), la fiabilidad (ofrecen el margen de error de la medida) y la validez (grado de adecuación al objeto medido). La rapide $z$ de su aplicación y corrección, por la comodidad, constituye también una cualidad práctica muy apreciada de las POs. Sin embargo, para alcanzar estas ventajas es necesaria una mayor preparación específica, en particular, un proceso más largo y meticuloso en la creación y diseño de cada prueba.

En el caso de los alumnos de COU, además de las calificaciones finales de curso, los alumnos llegan a la universidad tras haber superado también las pruebas de acceso (selectividad). A pesar de la duplicidad de los filtros, las limitaciones de la selectividad y las calificaciones finales del COU para cumplir una función de orientación fiable sobre la instrucción de los alumnos ha sido reconocida y demostrada (Escudero 1984, Aguirre
1984, Sánchez 1989, Muñoz, Navío, González y Munuera 1987), por lo que esas notas no son referentes válidos para diagnóstico. Todo ello ha generađo propuestas de selección alternativas, específicas para cada centro universitario (Groberna, López y Pastor 1987, Muñoz et al. 1987), o bien por área y tipo de estudios (Escudero 1987). Mitter (1979) ha demostrado que los sistemas de selección basados en tests o pruebas estandarizados tienen una capacidad orientadora para los alumnos, diagnóstica para los profesores y predictiva del rendimiento futuro, que resulta superior a otros sistemas; pero éste no es el modelo de evaluación escolar habitual en nuestro país, aunque recientemente se ha realizado una experienciapiloto de una evaluación con tests para la selectividad (ABC 1991, El País 1991).

También son escasas las investigaciones que emplean pruebas objetivas (POs). En una revisión exhaustiva de las investigaciones sobre rendimiento académico espa* ñolas, Álvaro (1990) indica que sólo el $13 \%$ considera las POs como instrumentos de investigación; si se tiene en cuenta que la mayoría de ellas se refieren a la Educación Básica, es imaginable, por extrapolación, la situa ción defícitaria en el BUP y COU (véase una revisión más amplia de estas últimas en Vázquez, 1989). Sin duda, el proyecto más importante, por su magnituđ, que ha empleado sistemáticamente POs como instrumento de evaluación ha sido la Evaluación Extema de la Refor- 
ma de las Enseñanzas Medias (González 1988, Marrero y Espino 1988); la baja relación obtenida ( $R$ múltiple .25 para la nota global frente a cuatro pruebas objetivas; correlación entre Matemática y PO de Cálculo - Ia más alta- .43) y el análisis de correlación canónica llevan a concluir que calificaciones y puntuaciones objetivas constituirían dos dimensiones diferenciadas del rendimiento escolar, con un sustrato común bajo. En otro estudio (Vázquez 1990a) referido a la Física y Química de Bachillerato, se apoya la tesis anterior que subraya las diferencias entre calificaciones y POs, por encima de su coincidencia, a pesar de que ambos, teóricamente, deberían medir las capacidades alcanzadas a través del aprendizaje escolar; la predicción del rendimiento académico muestra coefícientes de regresión bajos (Rs múltiple más elevadas .37 y .38 ).

Específicamente para el COU, Touron (1984) aplicó una Quede 37 ítems de Física a 106 alumnos de primero obteniendo una media de aciertos de $34 \%$, una correlación de .2203 con la calificación de Física final de curso y la fiabilidad de la PO que fue de .681 (KR20); Touron obtiene un rendimiento de $48 \%$ aplicando a un centenar de alumnos una PO de Química integrada sólo por ítems de Conocimiento, Comprensión y Aplícación con una correlación de .4832 con la califícación de Química de primero. También Escudero (1984) aplicó una PO general de conocimientos, no ofreciendo datos relativos a la Química o Física. Casanova, San José y Pérez (1985) aplicaron una prueba de Física de 54 ítems a 114 alumnos de primer curso de Físicas y de Químicas: la puntuación media de aciertos fue del $32 \%$ y sólo el $8 \%$ de los alumnos tuvieron más de un $50 \%$; las calificaciones al final del curso ( $55 \%$ de aprobados) les hace concluir que los estudios en la Universidad han seleccionado y heterogeneizado a los alumnos. Muñoz, Navío, González y Munuera (1987) evalúan conocimientos de Qúmica mediante una $\mathrm{PO}$, concluyendo que la distribución de las puntuaciones difiere de las obtenidas en la selectividad.

Por tanto, los estudios que relacionan calificaciones con POs concluyen que las varianzas comunes entre ambas variables son moderadas, muy por debajo de las que corresponden al rendimiento (calificación) anterior, y de las más moderadas para las variables de inteligencia o aptitudes, por citar algunas de las variables más empleadas y mejor operacionalizadas (Vázquez 1989). El problema que se plantea en este estudio es contrastar la baja relación existente entre las calificaciones finales de Química y Física de COU y en el primer curso universitario con las puntuaciones de sendas POs de Química y Física aplicadas a los alumnos. La hipótesis central pronostica una baja correlación; a la iuz de los resultados obtenidos, se discutirán las causas plausibles de los mismos.

\section{MÉTODO}

\section{Sujetos}

La PO de Física se aplicó a 455 alumnos matriculados en Física de primer curso de universidad en diversas espe- cialidades (Físicas/Ingenieros, Químicas, Biológicas, Informática y Magisterio) en los cursos 1986-87 (105) y $1989-90$ (350), 287 hombres y 141 mujeres, proviniendo de centros públicos (258) y privados (177) de COU; ia PO de Química se aplicó a 267 alumnos de la asignatura Química de primer curso en las licenciaturas de Ciencias (Químicas, Físicas y Biológicas), en los cursos 1987-88 (111 alumnos) y 1988-89 (156 alumnos), formada por 154 varones y 110 mujeres, de los cuales 142 habían cursado el COU en centros públicos y 112 en colegios privados.

\section{Instrumentos}

La PO de Física está formada por 30 ítems sobre contenidos del programa de Física de COU, todos con el mismo formato: cuestiones de elección múltiple con cinco alternativas de respuesta, de las cuales sólo una es correcta (cuatro distractores). Por construcción, los ítems están clasificados conforme a dos aspectos: la Categoría cognitiva y el Tema de contenido a que corresponde. Las Categorías empleadas clasifican los ítems según el nivel de exigencia cognitiva de cada uno, tomando como referencia los niveles taxonomizados por Bloom (1971): Comprensión (3), Aplicación (16), Análisis (5) y Síntesis (6). Comoes bien conocido, estos niveles están jerarquizados inclusivamente de más sencillo (Comprensión) a más complejo (Síntesis). Los Temas se corresponden con los contenidos definidos en el programa de Física de COU: Cálculo Vectorial (4), Dinámica (9), Trabajo-Energía (2), Rotación (2), Campos (7), Ondas (4), Corriente Alterna (2),

La PO de Química está formada por 40 ítems sobre conocimientos de Química contenidos en el programa de COU con el mismo formato que el anterior. Por construcción, los ítems de la PO aplicada están estructurados en Categorías cognitivas y Temas de contenidos. Las Categorías se corresponden con las taxonomizadas por Bloom (1971), y el número de ítems en cada una está graduado de acuerdo con el desarrollo evolutivo de los alumnos, predominando en este caso los ítems de las Categorías superiores: Conocimiento (3), Comprensión (8), Aplicación (11), Análisis (9), Śntesis (9). Los Temas son los contenidos del programa de Química de COU agrupados así: Leyes Generales de Química (8), Estructura atómica y Enlace Químico (6), Termoquímica y Equilibrio Químico (8), Reacciones químicas (12) y Química Orgánica (6).

\section{Procedimiento}

Los criterios empleados para la construcción y selección de los ítems de la PO intentan subrayar más las destrezas de razonamiento y resolución de cuestiones cortas, que tareas de simple recuerdo de información. La PO fue aplicada a los alumnos al comienzo del curso primero en la universidad, como si fuera el primer examen de la asignatura, para asegurar el interés y esfuerzo en su ejecución, disponiendo de 55 minutos para responder. 
Las puntuaciones en las variables de la PO se han obtenido asignando un punto a los ítems acertados y cero a los no acertados (o no contestados). Estas variables han sido las siguientes:

Globales de toda la PO: Aciertos (ítems acertados), Omisiones (ítems no contestados) y Errores (items equivocados).

Categorías cognitivas: número de ítems acertados en los ítems correspondientes a cada Categoría de las construidas.

Temas de contenidos: número de ítems acertados en los ítems correspondientes a cada Tema de los asignados por construcción.

Otras variables empleadas en este estudio son las calificaciones académicas de asignaturas de COU y primero de universidad, codificadas con las puntuaciones otorgadas en una escala numérica (10 a 99), o si están dadas cualitativamente, asi: No presentado / Muy deficiente (10), Insuficiente / Suspenso (30), Suficiente $(50,45)$, Bien $(65,60)$, Notable $(80,75)$, Sobresaliente $(95,90)$ y Matrícula de Honor (99), el primer valor si la calificación se ha obtenido en junio, y el segundo cuando se ha obtenido en septiembre.

\section{RESULTADOS}

Las características psicométricas de las $\mathrm{PO}$ y el proceso seguido para su validación empírica (confección, análisis de ítems, tipificación de puntuaciones, fiabilidad y validez) han sido presentadas en otro lugar (Vázquez 1990 c 1991) y serán objeto de una descripción más detalladá tanto en los aspectos teóricos como empíricos (Vázquez 1992c). Los coeficientes de fiabilidad ( $\alpha$ de Cronbach) son buenos (PO de Física .7296; PO de Química .7449) y los đatos sobre su validez (de contenido, externa y de constructo) son todos favorables, de manera que ratifican la aplicabilidad de las POs con los fines diagnósticos y de investigación para los que se han construido.

Las puntuaciones obtenidas en la PO de Física muestran un rendimiento de los alumnos deficiente: la puntuación media de Aciertos es baja 10.62 puntos (35\%), con desviación típica 4.15; la puntuación de Errores, 12.65 puntos (42\%) D.T. 5.50, es mayor que los Aciertos, existiendo una tasa muy alta de Omisiones de respuesta, 6.72 puntos (22\%) D.T. 6.23 (Vázquez 1992b). Las puntuaciones obtenidas en la PO de Química también muestran un bajo rendimiento de los alumnos: la puntuación de Aciertos es baja (media 14.03, 35.09\%; DS= 5.3 ; la puntuación media de Errores $(19.42,48.57 \%$; $\mathrm{DS}=7.4$ ) es significativamente mayor que los Aciertos, en tanto que la media de Omisiones $(6.53,16.34 \%$; DS= 7.9) también es relativamente alta (Vázquez 1992a).

La tabulación cruzada de las calificaciones de Física / Química de COU frente a las puntuaciones brutas de Aciertos en las POs, agrupadas en intervalos de 5 puntos, (aproximadamente una desviación típica) permite una primera aproximación al análisis de la relación existente entre calificaciones y puntuaciones en las POs (Tablas I y II). La prueba de chi-cuadrado para verificar la dependencia / independencia entre estas variables es significativa $(\mathrm{p}<.0000)$, por lo que la hipótesis de la independencia entre ambas variables debe ser rechazada: ambas variables están relacionadas significativamente. Sin embargo, cuando se analizan los contenidos de las celdas en esas tablas es evidente la mixtificación entre algunas de ellas y las situaciones excepcionales.

Tabla I

Tabulación cruzada de la puntuación de aciertos (escalada en intervalos de clase de 5 puntos) en la prueba objetiva Física-COU86 por la Calificación Anterior en Física de COU.

\begin{tabular}{|c|c|c|c|c|}
\hline \multicolumn{4}{|c|}{ Calificación de FisICA - COU } & \multirow{2}{*}{ Total } \\
\hline Suficiente & Bien & Notable & Sobresaliente & \\
\hline 0 a 5 puntos ...... 20 & 3 & 3 & & 26 \\
\hline$\%$ Tabla ........5,2 & 0,8 & 0.8 & & 6,8 \\
\hline$\%$ Col ...... 10,4 & 2,8 & 5,3 & & 6,8 \\
\hline$\%$ Fila ....... 76,9 & $\pm 1,5$ & 11,5 & & 100,0 \\
\hline 6 a 10 puntos ....97 & 44 & 16 & 3 & 160 \\
\hline$\%$ Tabia ... 25,3 & $\$ 1,5$ & 4,2 & 0,8 & 41,8 \\
\hline$\%$ Col..... 50,5 & 40,4 & 28,1 & 12,0 & 41,8 \\
\hline$\%$ Fila $\ldots \ldots . .60,6$ & 27,5 & 10,0 & 1,9 & 100,0 \\
\hline 11 a 15 puntos .. 64 & 43 & 28 & 12 & 147 \\
\hline \% Tabla ... 16,7 & 11,2 & 7,3 & 3,1 & 38,4 \\
\hline$\%$ Col. ..... 33,3 & 39.4 & 49,1 & 48,0 & 38,4 \\
\hline$\%$ Fila ....... 43,5 & 29,3 & 19,0 & 8,2 & 100,0 \\
\hline 16 a 20 puntos ...9 & 18 & 9 & 6 & 42 \\
\hline$\%$ Tabla, ... 2,3 & 4.7 & 2,3 & 1,6 & 11.0 \\
\hline$\%$ Col, ...... 4,7 & 16,5 & 15,8 & 24,0 & 11,0 \\
\hline$\%$ Fila ....... 21,4 & 42,9 & 21,4 & 14,3 & 100,0 \\
\hline 21 a 25 puntos ... 2 & 1 & 1 & 4 & 8 \\
\hline$\%$ Tabla ... 0,5 & 0.3 & 0,3 & 1,0 & 2,1 \\
\hline$\% \operatorname{Cot}, \ldots . . . \quad 1,0 \%$ & 0,9 & 1,8 & 16,0 & 2,1 \\
\hline$\%$ Fila ...... $25,0 \%$ & 12,5 & 12,5 & 50,0 & 100,0 \\
\hline Total ........... 192 & 109 & 57 & 25 & 383 \\
\hline Media ...... 11,8 & 13,6 & 14,0 & 17.2 & 13,0 \\
\hline DvSt ......... 3,9 & 4,0 & 4,2 & 4,6 & 4,3 \\
\hline
\end{tabular}

Para el caso de la Física (Tabla I) 6 alumnos con nota (Bien, Notable) no pasan de 5 aciertos en la PO; 3 alumnos calificados sobresalientes no pasan de 10 acier. tos; 2 alumnos calificados sólo como suficientes obtie. 
nen puntuaciones máximas en la PO... Sólo un $13 \%$ de alumnos consiguen una puntuación en la $\mathrm{PO}$ superior a la puntuación central del intervalo (15 puntos). La última fila contiene las puntuaciones en la PO; el grupo de sobresalientes tiene las diferencias más altas respecto a los demás.

\section{Tabla II}

Tabulación cruzada de las puntuaciones de Aciertos frente a las calificaciones en Química de COU.

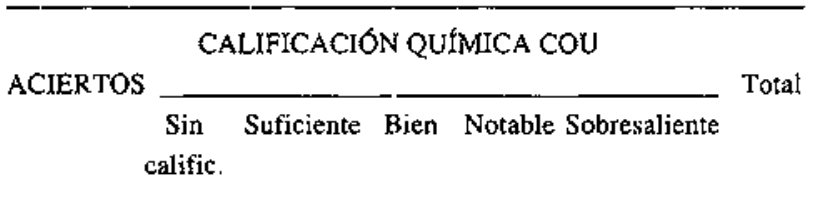

\begin{tabular}{|c|c|c|c|c|c|}
\hline Menos de $5 \ldots \ldots .4$ & 3 & 1 & & & 8 \\
\hline \% Tabla ........ 1,9 & 1,4 & 0.5 & & & 3,7 \\
\hline$\%$ Col..........25,0 & 4,2 & 1,5 & & & 3,7 \\
\hline$\%$ Fila ......... 50,0 & 37,5 & 12,5 & & & 100,0 \\
\hline 6 a $10 \ldots \ldots \ldots \ldots$ & 19 & 9 & 1 & 2 & 34 \\
\hline$\%$ Tabla ........1,4 & 8,9 & 4,2 & 0,5 & 0,9 & 15,9 \\
\hline$\% \mathrm{Col} \ldots \ldots . . .18,8$ & 26,8 & 13,8 & 3,1 & 6,7 & 15,9 \\
\hline \% Fila $\ldots \ldots \ldots \ldots . . .8,8$ & 55,9 & 26,5 & 2,9 & $\$, 9$ & 100,0 \\
\hline 11 a $15 \ldots \ldots \ldots$ & 31 & 29 & 15 & 9 & 90 \\
\hline \% Tabla ........ 2,8 & 14,5 & 13,6 & 7,0 & 4,2 & 42,1 \\
\hline \% Col.........37,5 & 43,7 & 44,6 & 46,9 & 30,0 & 42,1 \\
\hline$\%$ Fila .......... 6,7 & 34,4 & 32,2 & 16,7 & 10,0 & 100,0 \\
\hline 16 a $20 \ldots \ldots \ldots \ldots . .1$ & 15 & 18 & 7 & 9 & 50 \\
\hline \% Tabla ........ 0,5 & 7,0 & 8,4 & 3,3 & 4,2 & 23,4 \\
\hline$\% \mathrm{Col} \ldots \ldots \ldots \ldots, 3$ & 21,1 & 27,7 & 21,9 & 30,0 & 23,4 \\
\hline$\%$ Fila $\ldots \ldots \ldots . . .2,0$ & 30,0 & 36,0 & 14,0 & 18,0 & 100,0 \\
\hline 21 a $25 \ldots \ldots \ldots \ldots$ & 3 & 7 & 4 & 5 & 21 \\
\hline \% Tabla ......... 0,9 & 1,4 & 3,3 & 1,9 & 2,3 & 9,8 \\
\hline \% Col.........12,5 & 4,2 & 10,8 & 12,5 & 16,7 & 9,8 \\
\hline \% Fila ........... 9,5 & 14,3 & 33,3 & 19,0 & 23,8 & 100,0 \\
\hline 26 a $30 \ldots \ldots \ldots \ldots$ & & 1 & 5 & 4 & 10 \\
\hline$\%$ Tabla ........... & & 0,5 & 2,3 & 1,9 & 4,7 \\
\hline$\% \mathrm{Col} \ldots \ldots \ldots \ldots$ & & 1,5 & 15,6 & 13,3 & 4,7 \\
\hline$\%$ Fila .............. & & 10.0 & 50,0 & 40,0 & 100,0 \\
\hline 31 a $35 \ldots \ldots \ldots \ldots$ & & & & 1 & 1 \\
\hline$\%$ Tabla ........... & & & & $0,5 \%$ & $0.5 \%$ \\
\hline$\%$ Col $\ldots \ldots \ldots \ldots$ & & & & $3,3 \%$ & $0,5 \%$ \\
\hline$\%$ Fila .............. & & & & $100,0 \%$ & $100,0 \%$ \\
\hline Total $\ldots \ldots \ldots \ldots \ldots . . . . .16$ & 71 & 65 & 32 & 30 & 214 \\
\hline Media, ....... 13,1 & 14,7 & 16,8 & 19,5 & 20,5 & 16,8 \\
\hline Dv Std, , ......6,6 & 4,5 & 4,8 & 5,9 & 6,3 & 5,7 \\
\hline \% Tabla ....... 7,5 & 33,2 & 30,4 & 15,0 & 14,0 & 100,0 \\
\hline
\end{tabular}

La lectura de la tabla IF por las calificaciones, muestra que los alumnos que obtuvieron Suficiente en Química de COU, se distribuyen simétricamente entre los grupos de puntuaciones, los calificados con Bien y Notable tienen casi la mitad de alumnos en el grupo intermedio, y los Sobresalientes reúnen el $60 \%$ entre los dos grupos intermedios. Leyendo la tabla II por los grupos de puntuación en Aciertos, los grupos extremos (menos de 5 y más de 26) aparecen claramente relacionados con las puntuaciones bajas (Suficiente, Bien) y altas (Notable, Sobresaliente), respectivamente, pero entre ambos no llegan al $10 \%$ de la muestra. El grueso de los alumnos se reparte sobre los cuatro gupos de puntuaciones centrales, sesgado hacia los dos grupos más bajos. Las puntuaciones 6 a 10 están relacionadas mayoritariamente con los aprobados, en tanto que los dos grupos centrales ( 11 a 15 y 16 a 20) están distribuidos sobre todas las calificaciones con tasas paralelas a la tasas de la muestra total; el grupo de 2 I a 25 está ligeramente sesgado hacia las calificaciones más altas. La distancia entre los grupos de las distintas calificaciones en puntuaciones de la $\mathrm{PO}$ es bastante regular (última fila), siendo menor en los Sobresalientes.

Las correlaciones de las distintas variables de la PO con las calificaciones de las asignaturas consideradas (Tabla III) cuantifican mejor las relaciones entre las variables; Aciertos presenta las correlaciones más altas con las distintas asignaturas, que tienden a ser mayores con las asignaturas de primero que con la calificación de COU, tanto para la Química como para la Física, apuntando la posibilidad de que las POs tengan una mayor capacidad predictiva del rendimiento académico futuro que capacidad diagnóstica respecto a las calificaciones de COU. Errores y Omisiones no correlacionan tan significativamente, con tendencia a ser negativas.

Entre los Temas de Física, las variables más correlacionadas son Campos y Vectores, en tanto que C. Alterna y Ondas no correlacionan; entre las Categorias, la variable Análisis es la mejor correlacionada con las calificaciones. Nótese las elevadas correlaciones que llegan a alcanzar Álgebra, Cálculo y Física de primero entre sí, que casi doblan las correlaciones con las variables de las POs.

La correlación entre calificación de Física de COU y la puntuación de Aciertos es significativa $(.3373 ; \mathrm{p}<.01$ ), pero moderada y su cuadrado, que indica la tasa de varianza común a ambas variables, sería de un $11 \%$, baja. Las correlaciones de Física de COU con las asignaturas de primero son del mismo orden, siendo la de Álgebra la más alta.

Para la Química (mitad inferior de la tabla III), las Categorías con correlaciones más significativas son Comprensión, Aplicación y Análisis, y, entre los Temas, correlacionan significativamente con todas las asignaturas Átomos-Enlaces y Termoquímica-Equilibrio, en tanto que Química Orgánica no correlaciona significativamente. Con las naturales precauciones, ya que la significación del coeficiente de correlación entre dos variables depende del número de casos analizados, se observa que las correlaciones de las variables de la PO con respecto a la Quírnica de COU (pasadas) tienen una 
Tabla III

Correlaciones de las variables de la prueba objetiva Física-COU86/ Química-COU87 con las calificaciones anteriores de Física/Química de COU y posteriores (asignaturas de primer curso universitario).

\begin{tabular}{|c|c|c|c|c|c|}
\hline \multicolumn{2}{|c|}{$\mathrm{COU}$} & \multicolumn{4}{|c|}{ PRIMERO } \\
\hline PO FCOU.86 & & & & & \\
\hline & Física & Álgebra & Cálculo & Física & Química \\
\hline Casos $=$ & 383 & 67 & 138 & 140 & 133 \\
\hline ALGEBRA & $.4867 * *$ & & & & \\
\hline CÁLCULO & $.3205 * *$ & $.7030^{* *}$ & & & \\
\hline FÍSICA & $.3342 * *$ & $.5778^{* *}$ & $.6961 * *$ & & \\
\hline QUÍMICA & $.3713^{* *}$ & $.4959 * *$ & $.4681 * *$ & $.5546 * *$ & \\
\hline ACIERTOS & $.3373^{* *}$ & $.4484 * *$ & $.3027 * *$ & $.3880^{* *}$ & $.3216^{* *}$ \\
\hline OMISION & $-.1883^{* *}$ & -.3054 & -.1446 & -.1361 & -.2124 \\
\hline ERRORES & -.0403 &. .1427 & -.0950 & -.1807 &. .0320 \\
\hline VECTORES & $.2632 * *$ & $.3439 *$ & $.3138^{* *}$ & $.2863^{* *}$ & .1533 \\
\hline DINÁMICA & $.1431^{*}$ & .2218 & $.2223^{*}$ & $.2889 * *$ & .1421 \\
\hline TRABENER & $.1801^{* *}$ & .2280 & .0377 & .0923 & .0912 \\
\hline ROTACION & $.1657^{*}$ & $.3136^{*}$ & .1871 & $.2599^{*}$ & -.0255 \\
\hline CAMPOS & $.3059 * *$ & $.4497^{* *}$ & .2023 & $.2970^{* *}$ & $.4045^{* *}$ \\
\hline ONDAS & .1046 & .1764 & .0513 & .0686 & .1855 \\
\hline CALTERNA & .1284 & .0577 & .0473 & .0829 & .0383 \\
\hline COMPRENS. & $.2114^{* *}$ & .2942 & .1253 & .2035 & $.2610^{*}$ \\
\hline APLICACION & $.2821 * *$ & $.3853^{*}$ & .2160 & $.3229^{* *}$ & $.3251^{* *}$ \\
\hline ANÁLISIS & $.3245^{* * *}$ & $.3673^{*}$ & $.2593^{*}$ & $.2521^{*}$ & $.2305^{*}$ \\
\hline SINTESIS & $.1433 *$ & .3016 & $3101^{* *}$ & $.3393^{* *}$ & .0319 \\
\hline
\end{tabular}

COU PRIMERO

PO QCOU-87

Física Álgebra Cálculo Física Química

\begin{tabular}{llllll}
\hline CaSOS & 198 & 50 & 109 & 109 & 109 \\
OMISION & -.1689 & .0413 & .0736 & -.0573 & .1527 \\
ACIERTOS & $.4082^{* *}$ & $.5428^{* *}$ & $.3191^{* *}$ & $.3679^{* *}$ & $.4774^{* *}$ \\
ERRORES & -.1271 & $-.4435^{*}$ & $-.2892^{*}$ & .2016 & $-.4723^{* *}$ \\
PCORREGIDA & $.3922^{* *}$ & $.5554^{* *}$ & $.3513^{* *}$ & $.3657^{* *}$ & $.5375^{* *}$ \\
CONOC. & .0360 & .2513 & $.3126^{* *}$ & $.3136^{* *}$ & $.2517^{*}$ \\
COMPREN. & $.3744^{* *}$ & $.4025^{*}$ & $.3536^{* *}$ & $.2736^{*}$ & $.4558^{* *}$ \\
APLICACION & $.3594^{* *}$ & $.4340^{*}$ & .2247 & $.3091^{*}$ & $.3804^{* *}$ \\
ANÁLISIS & $.3294^{* *}$ & $.3723^{*}$ & .2200 & $.3164^{* *}$ & $.3634^{* *}$ \\
SINTESIS & .1706 & $.4065^{*}$ & .0643 & .0847 & .1655 \\
& & & & & \\
LEYES-QUIM & $.3090^{* *}$ & .2802 & $.2667^{*}$ & $.2817^{*}$ & $.3561^{* *}$ \\
ATOM-ENL. & $.3444^{* *}$ & $.4543^{* *}$ & $.2793^{*}$ & $.2588^{*}$ & $.4062^{* *}$ \\
TERMO-EQUIL. $.3061^{* *}$ & $.5125^{* *}$ & $.2758^{*}$ & $.3571^{* *}$ & $.4324 * *$ \\
REACCIONES & $.3030^{* *}$ & $.4379^{*}$ & .1559 & .1762 & $.2871^{*}$ \\
Q-ORGÁNICA & .1610 & .2952 & .1811 & $.2919^{*}$ & .2145
\end{tabular}

${ }^{*} \mathrm{p}<.1 \quad{ }^{* *} \mathrm{p}<.01$ magnitud similar a las calificaciones (futuras) de las asignaturas de primero, resultado ciertamente sorprendente si se tiene en cuenta que la PO versa sobre los contenidos del programa de COU.

$\mathrm{El}$ análisis de regresión permite distinguir la correlación global de un conjunto de variables (predictores) respecto a otra variable dependiente (criterio) y, además, contrastar la diferente potencia predictiva de aquéllos cuando compiten entre si. Como predictores se usan diferentes conjuntos de variables de la PO y como variables dependientes, las calificaciones de Física / Química de $\mathrm{COU}$ (anterior) y Física / Química de primero (posterior). Se han ensayado tres conjuntos diferentes de predictores (teniendo en cuenta que las variables sean independientes entre sî): las variables globales Aciertos y Ertores(Omisiones sería dependiente de éstas, por definición, por lo que no tiene sentido su inclusión), las Categorías y los Temas. Para cada conjunto, el procedimiento ha sido el siguiente: en primer Iugar se han entrado todas las variables del conjunto en la ecuación de regresión (método «Enter»), si satisfacen un criterio amplio (tolerancias), obteniéndose el máximo valor de la varianza predicha por cada conjunto; en segundo lugar se eliminan de la ecuación, paso a paso (método «stepwise backward»), selectivamente, las variables que no contribuyen significativamente a la varianza predicha medida a través del coeficiente «beta» (coeficiente estandarizado de regresión, $\mathrm{F}<.10$ ), permaneciendo sólo en la ecuación los predictores que satisfacen este criterio de significación.

Desde el punto de vista metodológico, se observa que las diferencias en el coeficiente de regresión múltiple entre el método «enter « y «stepwise» son mínimas, aportando el segundo una visión más parsimoniosa (menos predictores), que es más útil porque indica los predictores más potentes del criterio. La regresión sobre la Física de COU (Tabla IV, mitad superior) muestra que los predictores principales son Aciertos (para el primer conjunto), Análisis, Aplicación y Comprensión entre las Categorías, y Campos, Vectores y Trabajo-Energía entre los Temas. La regresión respecto a la Física de primero ofrece como predictores principales los Aciertos, Síntesis y Aplicación entre las Categorías, y, entre los Temas, Campos, Vectores y Rotación. Las principales diferencias entre las calificaciones en ambas asignaturas se producen en la predicción desde el conjunto de categorías, que respecto a la Física de COU hace intervenir las categorías de más bajo nivel de exigencia cognitiva (Análisis, Aplicación, Comprensión), en tanto que respecto a la Física de primero el predictor principal resulta ser la categoría de Síntesis, la de más alto nivel cognitivo.

Las magnitudes cuantitativas de la predicción, en particular la proporción de varianza común predicha $\left(\mathrm{R}^{2}\right)$ también es diferente: la Física de COU obtiene una varianza común con los predictores inferior a la de primero; aquélla con valores en el intervalo .11 a .15, y ésta entre .15 y .18 . En conjunto, estos valores obtenidos son moderados y resulta sorprendente que la Física de COU tenga valores inferiores a la Física de primero, si se tiene en cuenta que la PO está construida sobre el programa de COU. 
Tábla IV

Análisis predictivo (regresión múltiple) de las caifificaciones de las asignaturas de Física/Química-COU y Física/Química-Primero empleando como predictores diversos subconjuntos de las variables de la prueba objetiva Física-COU86 (los predictores están escritos en orden decreciente de su importancia relativa en la ecuación de regresión).

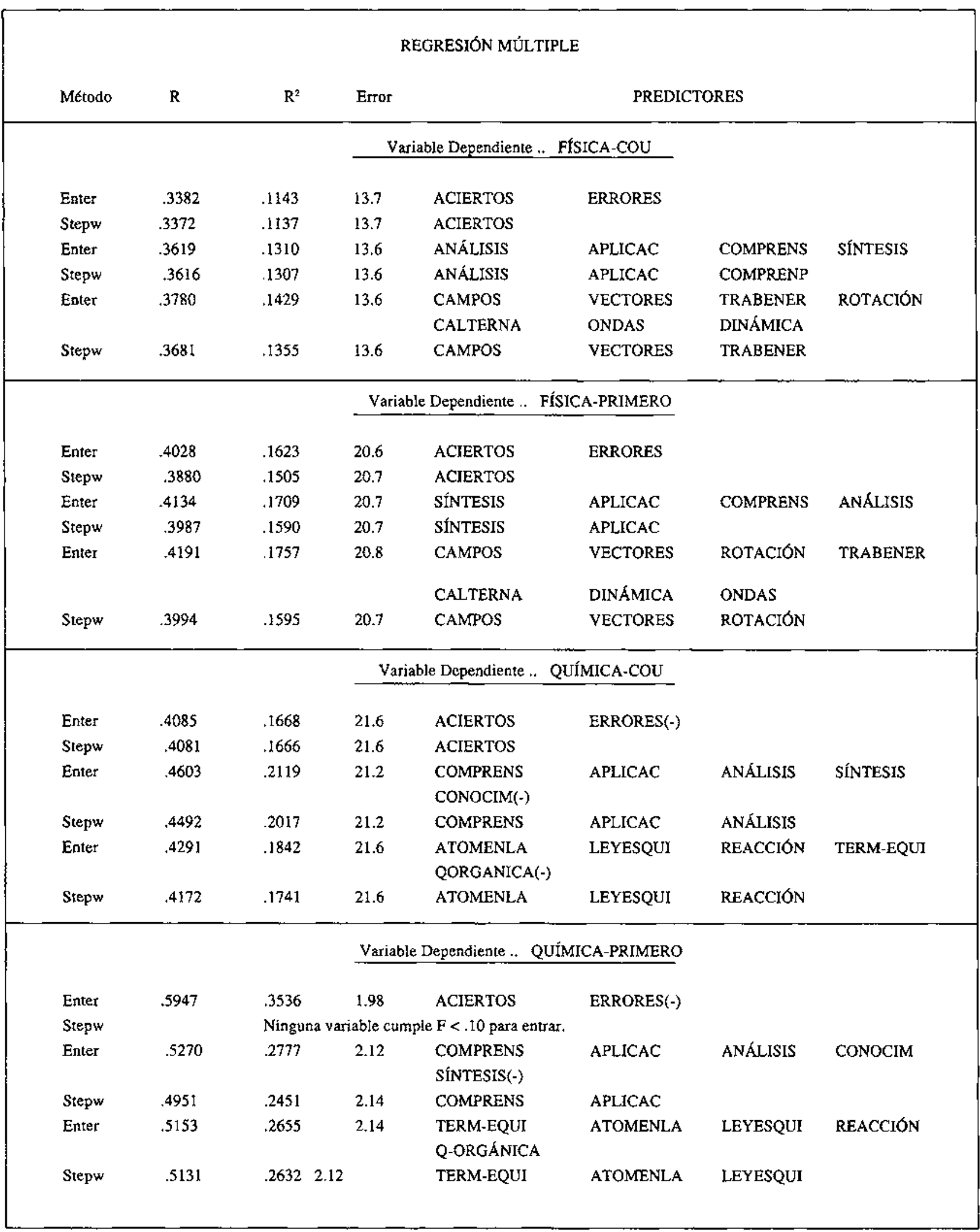


Análogamente, para la Química, los resultados (Tabla IV, mitad inferior) muestran que los predictores más importantes de la calificación obtenida en COU son, entre las Categorías, de mayor a menor, Comprensión, Aplicación y Arálisis, es decir, quedan excluidas las categorías extremas, la más sencilla jerárquicamente (Conocimiento) y la más compleja y alta en la jerarquía (Síntesis). Entre los Temas, Átomos-Enlace, Leyes Generales y Reacciones son los predictores mejores, siendo notable la exclusión de Termoquímica-Equilibrio, que era una de las variables bien correlacionada individualmente, ilustrando muy bien la diferencia entre estar bien correlacionada y aportar una varianza común con el criterio significativa, no explicada por los otros predictores con los que compite (condición de significación en la regresión múltiple). La variable Aciertoss es mejor predictor que Errores, y apoya el hecho habitual de considerar la puntuación global de aciertos de un test como la variable más significativa para representar la ejecución global del test. Para la regresión respecto a las calificaciones obtenidas en Física de primero, las diferencias más notables son la eliminación de la categoría de Análisis entre los predictores significativos y la aparición en primer lugar del tema de Termoquímica-Equilibrio y la desaparición de Reacciones.

Cuantitativamente, la mayor varianza común $\left(\mathrm{R}^{2}\right)$ criterio/predictores (proporción de la calificación de Química de COU explicada por los predictores usados) corres. ponde al grupo de variables de Categorías (.212), después el grupo de variables de Temas (.202), y las dos variables globales Aciertos / Errores (.167). Estos valores son inferiores en todos los casos a los obtenidos para las regresiones de la calificación de Química de primero $(.25, .26$ y .35 , respectivamente). Como en el caso de la Física, llama la altención que las variables de la PO explican más varianza de las calificaciones de Química de primero que de las calificaciones de Química de COU sobre cuyos contenidos versa la $\mathrm{PO}$.

Comparando los resultados de la regresión entre Física y Química para los distintos conjuntos de variables se observa que esta última posee varianzas explicadas mayores que aquélla, encontrándose los valores reflejados en la tabla IV en un intervalo entre .11 y .21 para las calificaciones de COU y entre .15 y .35 para las calificaciones de primero.

\section{DISCUSIÓN}

Las relaciones entre las puntuaciones de POs y calificaciones académicas son controvertidas; $s i$ ambas pretenden medir un mismo constructo, que podríamos llamar "estado instructivo», caracterizado por un cierto nivel de dominio, maestría o competencia en Física o Química debería esperarse una muy fuerte correlación entre las puntuaciones en la PO y las notas de Física / Química de COU. Sin embargo, aunque la correlación es significativa, los valores de la varianza común hallados mediante regresión son bajas en Física (inferiores a $15 \%$ ) y moderadas en Química ( $25 \%$ a $35 \%$ ), de modo que la mayor parte de la varianza común queda sin explicar. Por tanto, en la comparación entre las calificaciones emitidas por los profesores y las puntuaciones obtenidas de POs, para evaluar la instrucción en Física y Química de COU, los resultados muestran que son mucho más importantes las diferencias entre ellas que el sustrato común de ambas medidas del dominio.

La interpretación de estas diferencias y la explicación de Ios factores subyacentes tienen dos grandes vías. Por un lado, la investigación đocimológica, centrada en el análisis de los procesos evaluadores realizados por los profesores, resaitan la falta de validez y fiabilidad de la evaluación de los profesores, debido a la presencia de sesgos inevitables, por ser inherentes a la percepción humana (efectos halo, precedencia o contigúidad), que originan la aplicación (explícita o implícita) de criterios divergentes y variables. En este aspecto, la alternativa evaluadora de las POs, como instrumentos de diagnóstico rápidos, válidos y fiables, parece insustituible; además, las POs permiten: diagnosticar las concepciones alternativas valorando el grado de aprendizaje significativo (Treagust 1990, Vázquez $1990 \mathrm{~b}$ ), cumplimentar una importante función orientadora para los alumnos (sobre sus propias competencias y necesidades) y para los profesores (en relación con la programación de aula), e incluso ser instrumentos de aprendizaje (Foos y Fisher 1988). Las propuestas de diversas alternativas de estrategias de respuesta, como responder hasta llegar a la respuesta correcta (Frary 1982, Hutchinson y Barton 1987) o responder justificando la respuesta (Tamir 1990) mejoran e incrementan sustancialmente la calidad de la evaluación con POs. Ciertamente, el reconocimiento de estas virtudes de las POS no presupone que éstas deban ser los instrumentos únicos de evaluación; al contrario, una evaluación mixta (pruebas de ensayo, trabajos, observación de activiđades, pruebas objetivas, etc.) parece la estrategia de evaluación más apropiada.

Por otro lado, la investigación en didáctica de las ciencias centrada en el aprendizaje aporta otros factores explicativos de las diferencias mostradas, que resultan relevantes, por el consenso alcanzado.

En primer lugar, el concepto de aprendizaje significativo caracterizado por la retención comprensiva de los conceptos, integrados en la estructura mental del alumno en una red estable, amplia y multirrelacionada de significados, con un elevado valor funcional (útil), por tanto, susceptible de ser empleado en nuevas situaciones (Coll 1988) podría explicar también la baja correlación encontrada. La metodología de trasmisión / recepción de conceptos elaborados, predominante en la enseñanza / aprendizaje de las ciencias, caracterizada por el operacionalismo y la superficialiđad (Gil et al. 1988) conlleva además la inmediatez entre la transmisión y el examen evaluador, en cuyo éxito juegan un papel principal el efecto de la proximidad de la transmisión y la memoria a corto plazo. El resultado es que se refuerzan destrezas y cualidades poco significativas, que cumplen en un momento el papel asignado de repetición del conocimiento transmitido, pero están destinados a desaparecer con el paso del tiempo; así, Ios alumnos resuelven mejor problemas de tipo tradicional que conceptuales (Nurren- 
berg y Pickering 1987), e incluso resuelven problemas sin comprender los conceptos implicados en ellos (Lythcott 1990). Por ello, al cabo de unas semanas, cuando se realiza la evaluación con la $\mathrm{PO}$, muchos de los aprendizajes superficiales y memorísticos exhibidos por los alumnos en el momento đel examen han decaído drásticamente, de modo que lo que evalúa la $\mathrm{PO}$ está poco relacionado con lo evaluado en el examen tradicional; por tanto, la escasa varianza común entre calificaciones de COU y puntuaciones de las POs podría explicarse por la incapacidad de la metodología de transmisión para alcanzar aprendizajes significativos.

En segundo lugar, los estudios sobre la resolución de problemas muestran diversos factores relacionados con el procesamiento de la información y la estructuración conceptual de los conocimientos que dificutan el aprendizaje. Una buena organización conceptual en la memoria a largo plazo favorece el éxito en la resolución de problemas y viceversa (Kempa 1991); las ideas previas y el nivel formal en la estructuración conceptual determinan el rendimiento (Chandran et al. 1987), proponiéndose la necesidad de entrenamiento en estrategias intelectuales que favorezcan el procesamiento simultáneo de varias etapas y el razonamiento vertical (Pomés 1991). Cuando éstos factores no están desarrollados en los alumnos y éstos deben enfrentarse a tareas sencilias, pero que no están basadas en el simple recuerdo de información, sino que requieren el uso de estrategias de resolución, como los ítems de las POs, parece lógico encontrar un nivel más alto de fracaso, y además, una baja correlación con las calificaciones, ya que éstas, presumiblemente, no evaluaban estas estrategias, sino los algoritmos mecánicos típicos que la enseñanza receptiva ofrece para superar los exámenes, que además de no favorecer el desarrollo cognitivo (Pomés 1991), cuando falla el recuerdo, impiden llegar a la solución.

Estos mismos argumentos se refuerzan cuando se observa que las puntuaciones de las $\mathrm{POs}$ tienen una mayor potencia predictiva (respecto a las calificaciones futuras) que capacidad diagnóstica respecto al dominio en Física / Química (respecto a las calificaciones de COU). Si las relaciones con el rendimiento futuro (con el que no tendrían por qué tener relación significativa, en principio, porque no están diseñadas para medirlo) son significativas y superiores a las encontradas en las calificaciones de COU, se interpreta que las POs constituyen una medida de un núcleo de conocimientos significati-

\section{REFERENCIAS BIBLIOGRÁFICAS}

$A B C, 1991$. Selectividad experimental, una propuesta sin futuro. $A B C$ de la Educación, 4 junio 1991 .

AGUIRRE DE CÁRCER, I., 1987. La enseñanza secundaria de la física: una reforma necesaria, Revista Española de Física, $1(2)$, pp. 5-10. vos, estructurados establemente en la memoria a iargo plazo, y, por tanto, disponibles funcionalmente para ser utilizados en los nuevos aprendizajes y con una proyección significativa hacia el futuro.

En conclusión, este estudio demuestra la poca consistencia existente entre las calificaciones académicas recibidas por los alumnos en Física / Química de COU y sendas evaluaciones del dominio de los alumnos realizadas mediante POs (construidas sobre aspectos esenciaies de los programas de COU) al comenzar su primer curso en la Universidad. Paradójicamente, las puntuaciones de las POs tienen una mayor varianza común con las calificaciones de final del curso primero que con las de COU.

A la luz de las aportaciones de la investigación en didáctica de las ciencias se han interpretadoestos resultados como una muestra más de la incapacidad de la metodología de trasmisión / recepción para producir aprendizajes significativos (funcionales) y dotar a los alumnos de estrategias adecuadas (estables y adaptativas) para la resolución de problemas. Las implicaciones para la enseñanza / aprendizaje de estas materias parecen claras; si al cabo de un lapso de tiempo tan breve como el que media entre acabar el COU e incorporarse los alumnos a la Universidad queda un sustrato común tan pequeño con lo reflejado en las calificaciones de COU, debería reflexionarse sobre la necesidad de liegar al cambio con- ceptual y metodológico que constituyen el instrumento necesario para llegar a aprendizajes realmente significativos; el momento actual de reforma del sistema educativo, puede ser una oportunidad inmejorable. El cambio metodológico es una condición necesaria para llegar al cambio conceptual, pero es importante comprobar que ese cambio conceptual se ha alcanzado: las $\mathrm{POs}$ (normativas o criteriales) son instrumentos adecuados para contrastar el cambio producido.

\section{AGRADECIMIENTOS}

Eł autor agradece a toóos los profesores de Física y de Química de primero de la Universidad de las Islas Baleares la colaboracion prestada en la apticación de las pruebas a los alumnos, así como los provechosos comentarios de un revisor anónimo que han contribufdo a enriquecer este informe.
ÁLVARO PAGE, M. et al., 1990. Hacia un modelo causal del rendimiento académico. (Centro de Investigación, Documentación y Evaluación: Madrid).

BLOOM, B.S., 1971. Taxonomía de los objetivos de la educación. (El Ateneo: Buenos Aires). 
CASANOVA, J., SAN JOSÉ, R. y PÉREZ, P.J., 1985. Contribución al análisis del fracaso escolar en el primer curso de las facultades de ciencias, Revista de Educación, 278, pp. 99. 117.

CHANDRAN, S., TREAGUST, D.F. y TOBIN, K., 1987. The role of cognitive factors in Chemistry achievement, Journal of Research in Science Teaching, 24(2), pp. 145.160.

COLL, C., 1988. Significado y sentido en el aprendizaje escolar. Reflexiones en torno aI concepto de aprendizaje significativo, Infancia y Aprendizaje, 41, pp. 131-142.

EL PAÍS, 1991. Los riesgos del test. El Pais Educación, Año X, 146,18 junio 1991.

ESCUDERO ESCORZA, T., 1984. Condicionantes y capacidad predictiva de la selectividad universitaria, Revista de Educación, 273, pp. 139-164.

ESCUDERO ESCORZA, T., 1987. Buscando una mejor selección de universitarios, Revista de Educación, 283, pp. 249. 283.

FOOS, P.W. y FISHER, R.P., 1988. Using tests as learning opportunities, Journal of Educational Psychology, 88(2), pp. 179-183.

FRARY, R.B., 1982. A simulation study of reliability and validity of multiple-choice test scores under six responsescoring modes, Journal of Educational Statistics, 7(4), pp. 333-351.

GIL, D., MARTÍNEZ-TORREGROSA, J. y SENENT, F., 1988. El fracaso en la resolución de problemas de Física: Una investigación orientada por nuevos supuestos, Enseñanza de las Ciencias, 6(2), pp. 131-146.

GONZÁLEZ, A.J., 1988. Indicadores del rendimiento escolar. Relación entre pruebas objetivas y rendimiento, Revista de Educación, 287, pp. 31-54.

GOBERNA, M.A., LÓPEZ, M.A. y PASTOR, J.T., 1987. La prediccion del rendimiento como criterio para el ingrese en la universidad, Revista de Educación, 283, pp. 235-248.

HUTCHINSON, T.P. y BARTON, D.C., 1987. A mechanical reasoning test with answer-until-correct directions confirms a qualitative description of partial information, Research in Science \& Technological Education, 5(1), pp. 93-101.

KEMPA, R.F., 1991. Students learning difficulties in Science. Causes and possible remedies, Enseñanza de las Ciencias, 9(2), pp. 119-128.

LYTHCOTT, J., 1990. Problem solving and requisite knowledge of Chemistry, Journal of Chemical Education, 67(3), pp. 248-252.

MARREROHERNÁNDEZ,H. y ESPINOMORALES,O., 1988. Evaluación comparativa del poder predictor de las aptitudes sobre notas escolares y pruebas objetivas, Revista de Educación, 287, pp. $97-112$.
MITTER, W. (ed.), 1979. The Use of Tests and Interviews for Admission to Higher Education. (NFER Pub. Co.: Windsor),

MUÑOZ, E, NAVÍO, J.A., GONZÁLEZ, A.R. y MUNUERA, G. 1987. Consideraciones sobre las pruebas de acceso a la universidad a partix de algunos resultados estadísticos, $R e$. vista de Educación, 283, pp. 267-287.

NURRENBERG,S.C. y PICKERING, M., 1987. Concept learning versus problem solving: Is there a difference?, Journal of Chemical Education, 64(6), pp. 508-510.

POMÉS, J., 1991. La metodología de resolución de problemas y el desanollo cognitivo: un punto de vista postpiagetiano, Enseñanza de las Ciencias, 9(1), pp. 78-82.

SÁNCHEZ GÓMEZ, J.L. 1989. El azar y la selectividad, Revista Española de Física, 3(2), p. 79.

TAMIR, P., 1990. Justifying the selection of answers in multiple choice items, International Journal of Science Education, $12(5)$, pp. 563-573.

TREAGUST, D. F., 1988. Development and use of diagnostic tests to evaluate students' misconceptions in science, $\boldsymbol{I n}$ ternational Journal of Science Education, 10(2), pp. 159169.

TOURON FIGUEROA, J., 1984. Factores del rendimiento académico en la universidad. (EUNSA: Pamplona).

VÁZQUEZ ALONSO, A., 1989. Rendimiento en Bachillerato: Aptitudes y Atribución Causal. Análisis del rendimiento objetivo en Fisica y Química. Tesis doctoral, Universidad de las Islas Baleares.

VÁZQUEZ ALONSO, A., 1990a. Rendimiento académico y rendimiento objetivo en Física y Química de Bachillerato, Enseñanza, 8, pp. 145-164.

VÁZQUEZ ALONSO, A., 1990b. Concepciones alternativas en Física y Química de Bachillerato: Una metodología diagnóstica, Enseñanza de las Ciencias, 8(3), pp. 251-258.

VÁZQUEZ ALONSO, A., 1990c. Evaiuación objetiva en Química de alumnos que comienzan estudios universitarios. Resúmenes de las Comunicaciones a la XXIII Reunión Bienal de la Real Sociedad Española de Química (p. 150). Salamanca, 23-28 septiembre 1990.

VÁZQUEZ ALONSO, A., 1991. Valiđación empírica de una prueba objetiva de Física de COU. Libro de resúmenes de las Comunicaciones a la XXIII Reunión Bienal de la Real Sociedad Española de Física (Tomo I, p. 67), Valladolid, 23-27 septiembre 1991 .

VÁZQUEZ ALONSO, A., 1992a (en prensa). La Química de COU: una evaluación objetiva, Revista Española de Física.

VÁZQUEZ ALONSO, A., 1992b (en prensa). Evaluación objetiva de la Física de COU, Revista Española de Física.

VÁZQUEZ ALONSO, A., 1992c. Evaluación objetiva y concepciones alternativas. Quimicay física de COU. Manuscrito enviado para publicación. 


\section{APÉNDICE \\ Muestra de ítems de las pruebas objetivas.}

\section{PRUEBA OBJETIVA de FISICA-COU-86}

Una bola de masa $1 \mathrm{~kg}$ se mueve a $2 \mathrm{~m} / \mathrm{s}$ perpendicularmente contra una pared. Rebota con velocidad $1,5 \mathrm{~m} / \mathrm{s}$ en la misma direccion. La variación de la cantidad de movimiento de la bola en unidades del S.I. es:
A) Cero
B) 0,5
C) 1
D) 2
E) 3,5

Un cuerpo de masa $m$ recorre a velocidad constante, $v$, una circunferencia de radio $\mathbf{R}$. El trabajo que realiza la fuerza centrípeta en una vuelta vale:
A) $\frac{\mathrm{m} \cdot \mathrm{v}^{2}}{\mathrm{R}} \cdot 2 \cdot \pi \cdot \mathrm{R}$
B) Cero
C) $\frac{m \cdot v^{2}}{R} \cdot 2 \cdot R$
D) $\frac{m \cdot v^{2}}{R} \cdot R$
E) Nada de lo anterior

Dos masas $m$ y $m^{\prime}$ están separadás una distancia de $D$ metros. Se acercan las masas hasta una distancia $\mathrm{D} / 10 \mathrm{~m}$. El modulo de la fuerza gravitatoria en esta situación respecto a la primera:
A) Queda igual
B) Aumenta diez veces
C) Aumenta cien veces
D) Aumenta mil veces
E) Disminuye diez veces

La velocidad de propagación de la onda y $=1,5 \cdot \cos \pi\left(\frac{x}{3}-\xi\right)$,
en donde $x$ se expresa en metros y $t$ en segundos, es:
A) $3 \mathrm{~m} / \mathrm{s}$
B) $1,5 \mathrm{~m} / \mathrm{s}$
C) $1 / 3 \mathrm{~m} / \mathrm{s}$
D) $6 \mathrm{~m} / \mathrm{s}$
E) $3 / \pi \mathrm{m} / \mathrm{s}$

En un circuito de corriente alterna $I(t)=200 * \operatorname{sen}(200 * t+\pi / 4)$ y $V(t)=2000 \cdot \operatorname{sen}(200 * t+\pi / 4)$, podemos decir que:
A) Necesariamente sólo existe resistencia pura
B) Sólo existe resistencia e inductancia (L)
C) Sólo existe resistencia e capacitancia (C)
D) Puede ser con sólo resistencia, pero también con $R, L$ y $\mathrm{C}$.
E) Ninguna de las anteriores

\section{PRUEBA OBJETIVA de QUIMICA-COU-87}

Dadas las reacciones con sus respectivos cambios de entalpía:

$$
\begin{aligned}
& 2 \mathrm{Cr}+(3 / 2) \mathrm{O}_{2} \rightarrow \mathrm{Cr}_{2} \mathrm{O}_{3}(\mathrm{~s}) \Delta \mathrm{H}=-1130 \mathrm{~kJ} \\
& \mathrm{C}(\mathrm{s})+(1 / 2) \mathrm{O}_{2} \rightarrow \mathrm{CO}(\mathrm{g}) \quad \Delta \mathrm{H}=-110 \mathrm{~kJ}
\end{aligned}
$$

¿Cuánto vale el cambio de entalpía para la reacción:

$$
3 \mathrm{C}(\mathrm{s})+\mathrm{Cr}_{2} \mathrm{O}_{3}(\mathrm{~s}) \rightarrow 2 \mathrm{Cr}(\mathrm{s})+3 \mathrm{CO}(\mathrm{g}) ?
$$
A) $+800 \mathrm{~kJ}$
B) $-800 \mathrm{~kJ}$
C) $-1460 \mathrm{~kJ}$
D) $+1020 \mathrm{~kJ}$
E) $+1460 \mathrm{~kJ}$

Los nitratos calentados por encima de su punto de fusión se descomponen; los nitratos de los metales alcalinos dan nitritos y los de los demás metales dan los óxidos correspondientes. De las siguientes reacciones señala la que NO es correcta:
A) $\mathrm{NaNO}_{3}+$ calor $\rightarrow \mathrm{NaNO}_{2}+\mathrm{O}_{2}$
B) $\mathrm{Pb}\left(\mathrm{NO}_{3}\right)_{2}+$ calor $\rightarrow \mathrm{PbO}+\mathrm{NO}_{2}+\mathrm{O}_{2}$
C) $\mathrm{AgNO}_{3}+$ calor $\longrightarrow \mathrm{Ag}_{2} \mathrm{O}+\mathrm{NO}_{2}+\mathrm{O}_{2}$
D) $\mathrm{KNO}_{3}+$ calor $\rightarrow \mathrm{KNO}_{2}+\mathrm{O}_{2}$
E) $\mathrm{CsNO}_{3}+$ calor $\longrightarrow \mathrm{Cs}_{2} \mathrm{O}+\mathrm{NO}_{2}+\mathrm{O}_{2}$

Si se mezclan cantidades iguales de cada una de las siguientes disoluciones, ¿en qué mezcla cabe esperar que se obtenga un $\mathrm{pH}$, menor que 7 ?
A) $\mathrm{HCl} 1 \mathrm{M}$ y $\mathrm{KOH} 1 \mathrm{M}$
B) $\mathrm{H}_{2} \mathrm{SO}_{4} 0.5 \mathrm{M}$ y $\mathrm{KOH} 1 \mathrm{M}$
C) $\mathrm{HCl} 1 \mathrm{M}$ y $\mathrm{NH}_{4} \mathrm{OH} 1 \mathrm{M}$
D) $\mathrm{CH}_{3} \mathrm{COOH} 2 \mathrm{M}$ y $\mathrm{KOH} 2 \mathrm{M}$
E) $\mathrm{NH}_{4} \mathrm{OH} 1 \mathrm{M}$ y $\mathrm{CH}_{3} \mathrm{NH}_{2} 1 \mathrm{M}$

Entre las siguientes reacciones, señala aquélla en la que el ácido sulfúrico actúa como agente oxidante:
A) $\mathrm{H}_{2} \mathrm{SO}_{4}+\mathrm{CaO} \rightarrow \mathrm{CaSO}_{4}+\mathrm{H}_{2} \mathrm{O}$
B) $2 \mathrm{H}_{2} \mathrm{SO}_{4}+\mathrm{K}_{2} \mathrm{CO}_{3} \rightarrow 2 \mathrm{KHSO}_{4}+\mathrm{CO}_{2}+\mathrm{H}_{2} \mathrm{O}$
C) $\mathrm{H}_{2} \mathrm{SO}_{4}+\mathrm{NaNO}_{3} \rightarrow \mathrm{NaHSO}_{4}+\mathrm{HNO}_{3}$
D) $2 \mathrm{H}_{2} \mathrm{SO}_{4}+\mathrm{C} \rightarrow \mathrm{CO}_{2}+2 \mathrm{SO}_{2}+2 \mathrm{H}_{2} \mathrm{O}$
E) $\mathrm{H}_{2} \mathrm{SO}_{4}+\mathrm{NaOH} \rightarrow \mathrm{Na}_{2} \mathrm{SO}_{4}+2 \mathrm{H}_{2} \mathrm{O}$

¿Cuâi de las siguientes moléculas orgánicas, representadas por sus fómulas, tiene todos sus átomos de carbono en el mismo plano?
A) $\mathrm{CH}_{3}-\mathrm{CH}_{2}-\mathrm{CH}_{2}-\mathrm{CH}_{3}$
B) $\mathrm{CH}_{3}-\mathrm{CH}_{2}-\mathrm{C} \cong \mathrm{CH}_{3}$
C) $\mathrm{CH}_{2}=\mathrm{CH}-\mathrm{CH}_{2}-\mathrm{CH}_{3}$
D) $\mathrm{CH}_{3}-\mathrm{CH}=\mathrm{CH}-\mathrm{CH}_{3}$
E) $\mathrm{CH}_{2}=\underset{\mathrm{CH}}{\mathrm{C}}-\mathrm{CH}_{3}$

Si una disolución que es $10^{-5} \mathrm{M}$ respecto a los iones $\mathrm{CO}_{3}{ }^{2 \cdot}$ se

mezcla con un volumen igual de otra disolucion $10^{-5} \mathrm{M}$ de un metal del grupo II, ¿cuál de los siguientes carbonatos precipiŁarấ?
A) $\mathrm{MgCO}_{3} \quad \mathrm{~K}_{\mathrm{p}} \mathrm{s}=1.1 \cdot 10^{-5} \mathrm{~mol}^{2} \cdot \mathrm{dm}^{.6}\left(298^{\circ} \mathrm{K}\right)$
B) $\mathrm{CaCO}_{3} \mathrm{~K}_{\mathrm{p}} \mathrm{s}=5.0 \cdot 10^{-9} \mathrm{~mol}^{2} \cdot \mathrm{dm}^{-6}\left(298^{\circ} \mathrm{K}\right)$
C) $\mathrm{SrCO}_{3} \quad \mathrm{~K}_{\mathrm{p}} \mathrm{s}=1.1 \cdot 10^{\cdot 10} \mathrm{~mol}^{2} \cdot \mathrm{dm}^{6}\left(298^{\circ} \mathrm{K}\right)$
D) $\mathrm{BaCO}_{3} \quad \mathrm{~K}_{\mathrm{p}} \mathrm{s}=5.5 \cdot 10^{10} \mathrm{~mol}^{2} \cdot \mathrm{dm}^{6}\left(298^{\circ} \mathrm{K}\right)$
E) Ninguno de los anteriores. 